\title{
Theory of optical excitations in dipole-coupled hybrid molecule-semiconductor layers: Coupling of a molecular resonance to semiconductor continuum states
}

\author{
Eike Verdenhalven, ${ }^{*}$ Andreas Knorr, and Marten Richter \\ Institut für Theoretische Physik, Nichtlineare Optik und Quantenelektronik, \\ Technische Universität Berlin, Hardenbergstr. 36, 10623 Berlin, Germany \\ Bjoern Bieniek and Patrick Rinke \\ Fritz-Haber-Institut der Max-Planck-Gesellschaft, Faradayweg 4-6, D-14195 Berlin, Germany \\ (Received 3 April 2014; revised manuscript received 21 May 2014; published 19 June 2014)
}

\begin{abstract}
We theoretically investigate the optical absorption of a hybrid system consisting of an organic molecular film on top of a semiconductor substrate. The electronic states of the isolated spatially separated constituents couple due to the Coulomb interaction of the optically induced charge carriers across the film-substrate interface. Focussing on the coupling of optical active molecular transitions to semiconductor continuum states, we find that the nonradiative dipole-dipole energy transfer causes the formation of coupled excitations, effectively reducing the excitation energy of the optical resonance in the molecular film and inducing a broadening of the associated absorption peak. In the framework of the Heisenberg equation of motion technique, we derive the Bloch equations for these hybrid systems. The input parameters for our model system of ladder-type quarterphenyl (L4P) molecules on the $\mathrm{ZnO}(10 \overline{1} 0)$ surface are taken from density functional theory calculations.
\end{abstract}

DOI: 10.1103/PhysRevB.89.235314

\section{INTRODUCTION}

Hybrid organic-inorganic systems have the potential to overcome the limitations of their individual components and to exhibit new functionality [1-3]. To improve the performance of optoelectronic devices, for example, the high charge-carrier mobility of inorganic semiconductors could be combined with suitably synthesized photoactive molecules [4] with wavelength-taylored light emission and absorption spectra. Moreover, pronounced nonlinear optical effects could emerge when Wannier excitons from the inorganic semiconductor side mix with the Frenkel excitons typically found in organic materials $[5,6]$. Due to recent advances in fabrication techniques, the first experimental signatures of nonradiative energy transfer processes between $\mathrm{ZnO}$ and conjugated molecules have been observed [7,8]. However, experimentally, it is difficult to determine the precise excitation transfer mechanism, knowledge of which would open up pathways for optimizing the efficiency of future devices.

In this paper, we focus on the theoretical analysis of energy transfer without electronic wave-function overlap, i.e., Förster processes between the components of the hybrid. Here, two possible resonant energy transfer paths are possible: coupling of molecular excitations (i) to semiconductor excitons (bound electron-hole pairs) or (ii) to continuum states (unbound electron-hole pairs) of the semiconductor. The first pathway resembles a molecular Förster transfer, but requires a close energetic match of the transition energies. In contrast, the second pathway relaxes this matching restriction since the electron-hole continuum of the semiconductor offers a broad range of transition energies for energetic coupling. If the coupling mechanism is sufficiently strong, pathway two would provide an efficient channel for exciting the molecular layer

\footnotetext{
*eikev@itp.tu-berlin.de
}

PACS number(s): 78.40.-q, 78.67.-n, 78.20.Bh

through external electrical pumping of the semiconductor layer by well established methods.

Previous theoretical work focused on the excitation transfer between Frenkel excitons in the organic layer and Wannier excitons in the substrate quantum well [6]. New, joint excitonmolecular polariton states at the interface of both components are predicted to mediate the exciton-exciton interaction of the semiconductor and the molecular film. While it is conceivable that this mechanism provides a strong coupling, it is restricted by the exact tuning of the transition energies of both components of the hybrid system, which may be difficult to achieve in experiments.

In this work, we investigate the coupling of molecular states to electronic continuum states that lie energetically above the semiconductor excitons (i.e., the aforementioned case ii). Such a coupling scheme describes the scenario of electrically pumping the semiconductor substrate. The free electron-hole gas that forms provides the energy for the optical emission of the molecular states. Likewise, in a photovoltaic application, the organic film would capture the incident light, whose energy is then transferred into semiconductor continuum states across the interface. It has recently become possible to investigate excitation transfer from first principles [9-13]. These approaches are based on time-dependent density-functional theory and propagate the Kohn-Sham equations in real time [9-13]. The motion of the nuclei is treated classically. To capture charge transfer excitations, which will be relevant for future work, range-separated exact-exchange kernels are used. However, these kernels are computationally expensive and limit the approach in the tractable system size.

In this paper, we develop a microscopic model for the nonradiative Förster coupling [14] between an organic molecular layer on a well defined surface of a quasi-twodimensional inorganic semiconductor. Our approach is based on a Heisenberg equation of motion technique $[15,16]$ to derive the optical many-body Bloch equations of the hybrid system in the Hartree-Fock limit $[17,18]$. This approach is 
computationally less expensive than the aforementioned fully time resolved first-principles calculations, but retains the microscopic description of the charge carrier dynamics, if parameterized with first-principles input. Although the present paper only addresses the effects of the Coulomb interaction in the limit of linear optics, our framework allows us to investigate more complex effects such as charge carrier relaxation and phonon-assisted charge transfer in future work.

Model Hamiltonian studies for Förster energy transfer are common for molecules, molecular aggregates and (functionalized) nanoclusters [19-27]. We here exploit the periodicity of both the inorganic substrate and the molecular layer to simplify the optical Bloch equations. We treat the organic layer by assuming a strictly lattice periodic arrangement of molecules with a periodicity different from the substrate lattice. This periodicity assumption allows us to treat the organic layer in a quasimomentum representation similar to the substrate. When taking into account the Coulomb interaction across the hybrid interface, the different lattices lead to selection rules in the Hamilton operator. In this work, we assume the organic layer to be weakly bonded to the inorganic substrate (i.e., no chemical bonds or hybridized states form at the interface). This allows us to clearly distinguish the contribution of the individual components in the optical spectrum. We parametrize our model with input from density-functional theory (DFT) calculations for ladder-type quarterphenyl (L4P) molecules on the $\mathrm{ZnO}(10 \overline{1} 0)$ surface. By solving the equations of motion, we calculate linear optical absorption spectra and examine the effect of the Coulomb interaction as a function of the system's geometry and transition dipole strength.

The article is structured as follows. First, we present the model system and develop a theoretical description, introducing the Hamilton operator. Then, we present numerical calculations based on input parameters obtained from DFT calculations for $\mathrm{L} 4 \mathrm{P}$ molecules on the $\mathrm{ZnO}(10 \overline{1} 0)$ surface.

\section{MODEL SYSTEM}

Our structural model consists of an inorganic semiconducting substrate that is covered by a noncovalently attached single layer of organic molecules (see Fig. 1). The unit cell of the substrate is given by the surface structure. For simplicity, we also assume the molecules to arrange in a periodic lattice with unit vectors parallel to the substrate surface cell. In reality, the organic film may be disordered or amorphous, which potentially weakens the coupling strength. The explicit inclusion of disorder effects will be the subject of future work. In this work we investigate how the Förster transfer mechanism between the semiconductor and the organic molecules is influenced by the geometry of the hybrid structure. The hybrid system is characterized by (i) the binding distance $\Delta z$ between the molecular film and the substrate, (ii) the

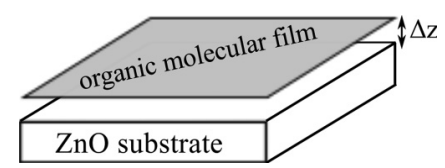

FIG. 1. Simplified sketch of the model system. $\Delta z$ gives the distance of the molecular film to the inorganic substrate. orientation of the molecular lattice relative to the substrate lattice, and (iii) the molecular coverage $\frac{N_{\mathrm{m}}}{N_{\mathrm{c}}}$ (i.e., the number of molecules per surface unit cell, where $N_{\mathrm{m}}$ and $N_{\mathrm{c}}$ are the number of molecular and surface unit cells, respectively). By varying these parameters within reasonable ranges around the equilibrium parameters obtained from our DFT calculations, we will illustrate their influence on the Förster transfer mechanism and the optical absorption of the hybrid material.

\section{OPTICAL SPECTRA}

We obtain linear optical absorption spectra by calculating the frequency-dependent total absorption coefficient $\alpha_{\text {tot }}(\omega)=$ $\alpha_{\mathrm{m}}(\omega)+\alpha_{\mathrm{sc}}(\omega)$. Here, $\alpha_{\mathrm{m}}(\omega)$ is the absorption coefficient of the molecular film in the presence of the semiconductor substrate and $\alpha_{\mathrm{sc}}(\omega)$ that of the semiconductor in the presence of the molecular layer [28]. Both $\alpha_{\mathrm{m}}$ and $\alpha_{\mathrm{sc}}$ are proportional to their respective susceptibilities, which connect the incident field and the resulting macroscopic polarizations,

$$
\hat{P}_{\mathrm{m} / \mathrm{sc}}(\omega)=\varepsilon_{0} \chi_{\mathrm{m} / \mathrm{sc}}(\omega) \hat{E}(\omega) .
$$

The total optical absorption coefficient $\alpha_{\text {tot }}(\omega)$ is proportional to the imaginary part of the total susceptibility, which is obtained by normalizing the total macroscopic polarization to the incident electrical field,

$$
\alpha_{\text {tot }}(\omega) \propto \omega \operatorname{Im} \chi_{\text {tot }}(\omega)=\omega \operatorname{Im}\left[\frac{\hat{P}_{\mathrm{m}}(\omega)+\hat{P}_{\mathrm{sc}}(\omega)}{\varepsilon_{0} \hat{E}(\omega)}\right] .
$$

In this paper, the optical response of the semiconductor substrate $\mathbf{P}_{\mathrm{sc}}(t)$ and the molecular layer $\mathbf{P}_{\mathrm{m}}(t)$ are evaluated in the far field limit, which is obtained by averaging over the microscopic scale:

$$
\begin{aligned}
& \mathbf{P}_{\mathrm{sc}}(t)=\frac{2 e_{0}}{V} \sum_{\mathbf{k}} \operatorname{Re}\left[\mathbf{d}_{\mathbf{k}}^{\mathrm{vc}} \sigma_{\mathrm{vc}}^{\mathbf{k k}}\right], \\
& \mathbf{P}_{\mathrm{m}}(t)=\frac{2 e_{0}}{V} \sum_{\mathbf{l}} \operatorname{Re}\left[\begin{array}{ll}
\mathbf{d}_{H L} & \sigma_{H L}^{\mathrm{ll}}
\end{array}\right] .
\end{aligned}
$$

Here, $\mathbf{k}$ and $\mathbf{l}$ denote the two-dimensional wave vectors parallel to the hybrid interface. The corresponding sums extent over the Brillouin zones associated to the lattice structure of the substrate and molecular adlayer. The volume of the entire system is given by $V$. The resulting expressions (3) and (4) link the macroscopic observables $\mathbf{P}_{\mathrm{sc} / \mathrm{m}}$ to the microscopic expectation values of the electronic transition amplitudes $\sigma_{\mathrm{vc}}^{\mathbf{k k}}(t)$ and $\sigma_{H L}^{\mathrm{ll}}(t)$. In the next section, we will derive equations of motion for the time dependence of these operators and show how the dynamics is influenced by many-body interactions in the Förster transfer limit.

\section{THEORETICAL APPROACH}

Our microscopic approach is based on a Heisenberg equation of motion technique $[15,16]$. In the Heisenberg picture, the equation of motion for an operator $O$ is given by $\mathrm{i} \hbar \frac{\mathrm{d}}{\mathrm{d} t} O=[O, H]$. The annihilation (creation) operators $a^{(\dagger)}$ create electrons or holes in the semiconductor or the molecule. The indices $A_{v}$ and $B_{v}$ represent the quantum mechanical states of the $v$ th molecule. These can be either the highest occupied molecular orbital (HOMO) abbreviated here by $H$ or the lowest 
unoccupied molecular orbital (LUMO) abbreviated by $L$. The semiconductor states are characterized by a two-dimensional wave vector $\mathbf{k}$ and band index $\lambda \in\{\mathrm{v}, \mathrm{c}\}$, where the wave vectors $\mathbf{k}$ correspond to the in-plane direction (i.e., $\mathbf{k}=\mathbf{k}_{\|}$).

\section{A. Hamilton operator}

The Hamilton operator in our approach is defined as follows:

$$
\begin{gathered}
H=H_{\mathrm{m}}+H_{\mathrm{sc}}+H_{\mathrm{e}-1}+H_{\mathrm{int}}, \\
H_{\mathrm{m}}=\sum_{A, v} E_{A_{v}} a_{A_{v}}^{\dagger} a_{A_{v}}, \quad H_{\mathrm{sc}}=\sum_{\mathbf{k}, \lambda} E_{\lambda}^{\mathbf{k}} a_{\mathbf{k}, \lambda}^{\dagger} a_{\mathbf{k}, \lambda}, \\
H_{\mathrm{e}-1}=\sum_{\substack{\mathbf{k} \neq \lambda^{\prime} \\
\mathbf{d}_{\lambda, \lambda^{\prime}}^{\mathbf{k}}}} \mathbf{E}(t) a_{\mathbf{k}, \lambda}^{\dagger} a_{\mathbf{k}, \lambda^{\prime}}+\sum_{\substack{A \neq B \\
v}} \mathbf{d}_{A_{v}, B_{v}} \cdot \mathbf{E}(t) a_{A_{v}}^{\dagger} a_{B_{v}}, \\
H_{\mathrm{int}}=\sum_{\substack{\mathbf{k}, \mathbf{k}^{\prime} \\
v}}\left(V_{\mathbf{k}^{\prime} \mathrm{c} H_{v}}^{\mathbf{k} v L_{v}} a_{\mathbf{k v} v}^{\dagger} a_{L_{v}}^{\dagger} a_{H_{v}} a_{\mathbf{k}^{\prime} \mathrm{c}}+V_{\mathbf{k}^{\prime} \mathrm{v} L_{v}}^{\mathbf{k c} H_{v}} a_{\mathbf{k c}}^{\dagger} a_{H_{v}}^{\dagger} a_{L_{v}} a_{\mathbf{k}^{\prime} \mathrm{v}}\right) .
\end{gathered}
$$

$H_{\mathrm{m}}$ and $H_{\mathrm{sc}}$ are the Hamiltonians for an isolated molecule and the semiconductor, respectively. $H_{\mathrm{e}-1}$ describes the interaction of the quantized charge carriers with an external classical light field (7) and $H_{\text {int }}$ the Coulomb interaction between the electrons in the substrate and the molecule.

$E_{A_{v}}$ and $E_{\lambda}^{\mathbf{k}}$ are the quasielectron and quasihole energies for the molecules and the semiconductor, respectively. These should, in principle, be computed in a quasiparticle formalism like the $G W$ approach [29-31]. Here, we approximate them with the DFT energies using a hybrid functional. The quasiparticle energies of the substrate are given by the band structure $E_{\lambda}^{\mathbf{k}}$ from which we include only one valence and one conduction band, which is sufficient for the resonant situation in $\mathrm{ZnO}$ considered here. We assume that the molecules are spatially well separated so that the interaction between them becomes negligible. The formation of molecular bands in more densely packed films will be considered in future work. Here, we take $E_{H}$ and $E_{L}$ to be equal for all molecules and adjust their values to lie just above the band edges of the two band semiconductor substrate. This choice yields maximal Förster transfer since the transfer rate decays with the difference between the HOMO-LUMO gap and the band gap of the semiconductor. In reality, the inorganic substrate and the molecules in the organic film have to be chosen such that this condition is fulfilled as closely as possible to obtain maximal Förster transfer. Since the molecular states are chosen to be in resonance with continuum states we can neglect the formation of bound semiconductor excitons.

In Eq. (7), the light-matter interaction is treated using a semiclassical approach in the dipole approximation [28] for both components of the hybrid material. The interaction strength is governed by the transition dipole moments $\mathbf{d}_{H L}$ and $\mathbf{d}_{\mathrm{vc}}$ for the molecules and the substrate, respectively. The incoming light is described by the classical electrical field $E(t)$. The Coulomb interaction is treated microscopically and fully quantized so that we can explicitly include Förster transfer process by means of the corresponding matrix elements $V_{\mathbf{k}^{\prime} \lambda^{\prime} B_{\nu}}^{\mathbf{k} \lambda A_{v}}$, which are discussed and defined below. In principle, within our approach, it is also possible to include the coupling to molecular and lattice vibrations.

\section{B. Transformation to momentum representation}

Up to this point we have treated the electrons of the semiconductor in a Bloch basis but used localized states for molecules. To obtain a consistent description, we have to transform the molecular operators to a Bloch momentum representation [32], too. In this work, we assume a lattice periodic arrangement of molecules. This choice corresponds to the best coupling limit and facilitates the construction of a microscopic model for charge carrier dynamics in hybrid materials. The idealized model derived in this work may serve as a starting point for the description of more realistic scenarios, including, e.g., disordered adlayers.

We assign each Bloch-based operator a two-dimensional wave vector $\mathbf{l}$. The transformation pair

$$
\begin{aligned}
& a_{A, \mathbf{l}}=\sum_{v}^{N_{m}} \frac{1}{\sqrt{N_{\mathrm{m}}}} e^{\mathrm{i} \mathbf{l} \cdot \mathbf{R}_{v}} a_{A_{v}}, \\
& a_{A_{v}}=\sum_{\mathbf{I}} \frac{1}{\sqrt{N_{\mathrm{m}}}} e^{-\mathrm{i} \mathbf{l} \cdot \mathbf{R}_{v}} a_{A, \mathbf{l}}
\end{aligned}
$$

is then unambiguously defined, if the wave vector $\mathbf{l}$ is restricted to the first Brillouin zone of the molecular lattice. To fulfill the periodic in-plane boundary conditions, the ratio of the total number of molecules $N_{\mathrm{m}}$ to the total number of unit cells $N_{\mathrm{c}}$ in the sample must be exactly the unit cell volume $V_{\mathrm{c}}$ divided by the molecular unit cell volume $V_{\mathrm{m}}$ (i.e., $N_{\mathrm{m}} / N_{c}=$ $\left.V_{\mathrm{c}} / V_{\mathrm{m}}\right)$. Assume that vectors $\tilde{\mathbf{a}}_{1}$ and $\tilde{\mathbf{a}}_{2}$ span the unit cell of the molecular layer. We can then find a reciprocal lattice spanned by the vectors $\tilde{\mathbf{b}}_{1}$ and $\tilde{\mathbf{b}}_{2}$ such that $\tilde{\mathbf{a}}_{i} \cdot \tilde{\mathbf{b}}_{j}=2 \pi \delta_{i, j}$. For a periodical arrangement of molecules, each molecular position $\mathbf{R}_{v}$ is given by

$$
\mathbf{R}_{v}=n_{1}^{v} \tilde{\mathbf{a}}_{1}+n_{2}^{v} \tilde{\mathbf{a}}_{2} \quad n_{1}^{v}, n_{2}^{v} \in \mathbb{Z},
$$

where $n_{1}^{v}$ and $n_{2}^{v}$ are integers. For a sufficiently large sample (and therefore large $N_{m}$ ), we can approximate:

$$
\sum_{v} \frac{1}{N_{m}} e^{\mathrm{i} \mathbf{Q} \cdot \mathbf{R}_{v}} \approx \sum_{m_{1}, m_{2}} \delta_{\mathbf{Q}, m_{1} \tilde{\mathbf{b}}_{1}+m_{2} \tilde{\mathbf{b}}_{2}} \equiv \sum_{\mathbf{G}} \delta_{\mathbf{Q}, \mathbf{G} .}
$$

With Eqs. (9) and (10) and a periodic arrangement of molecules the transformed Hamilton operator reads

$$
\begin{gathered}
H_{\mathrm{m}}=\sum_{A} E_{A} \sum_{\mathbf{l}} a_{A, \mathbf{l}}^{\dagger} a_{A, \mathbf{l}}, \quad H_{\mathrm{sc}}=\sum_{\mathbf{k}, \lambda} E_{\lambda}^{\mathbf{k}} a_{\mathbf{k}, \lambda}^{\dagger} a_{\mathbf{k}, \lambda}, \\
H_{\mathrm{e}-1}=\sum_{\substack{\mathbf{k} \\
\lambda \neq \lambda^{\prime}}} \mathbf{d}_{\lambda, \lambda^{\prime}}^{\mathbf{k}} \cdot \mathbf{E}(t) a_{\mathbf{k}, \lambda}^{\dagger} a_{\mathbf{k}, \lambda^{\prime}}+\sum_{A B} \mathbf{d}_{A, B} \cdot \mathbf{E}(t) \sum_{\mathbf{l}} a_{A, \mathbf{l}}^{\dagger} a_{B, \mathbf{l}}, \\
H_{\mathrm{int}}=\frac{1}{\mathcal{A}_{\mathrm{c}} N_{\mathrm{c}}} \sum_{\substack{\mathbf{k}, \mathbf{k}^{\prime} \\
\mathbf{l}, \mathbf{I}^{\prime}}} \sum_{\mathbf{G}} \delta_{\mathbf{I}^{\prime}-\mathbf{l}, \mathbf{k}^{\prime}-\mathbf{k}+\mathbf{G}}\left(\mathcal{V}_{\mathbf{k}^{\prime} \mathrm{c} H}^{\mathbf{k v} L} a_{\mathbf{k} \mathbf{v}}^{\dagger} a_{L, \mathbf{l}}^{\dagger} a_{H, \mathbf{I}^{\prime}} a_{\mathbf{k}^{\prime} \mathrm{c}}\right. \\
\left.+\mathcal{V}_{\mathbf{k}^{\prime} \mathrm{c} H}^{\mathbf{k v} L} a_{\mathbf{k} \lambda}^{\dagger} a_{A, \mathbf{l}}^{\dagger} a_{B, \mathbf{I}^{\prime}} a_{\mathbf{k}^{\prime} \lambda^{\prime}}\right) .
\end{gathered}
$$


From now on, we distinguish between wave vectors that reside in different Brillouin zones. Sums over $\mathbf{k}$ or $\mathbf{k}^{\prime}$ add up all wave vectors from the first Brillouin zone of the semiconductor substrate, whereas sums over $\mathbf{l}$ or $\mathbf{l}^{\prime}$ sum vectors from the first Brillouin zone of the molecular layer. The sum over G, stemming from Eq. (12), runs over all possible linear combinations of the molecular reciprocal lattice vectors. The corresponding Kronecker $\delta$ yields a selection rule for the momentum transfer. As we will explain later, this selection rule depends on the geometry of the components and governs the effect of the Coulomb interaction. In Eq. (15), we also redefined the Coulomb matrix element by extracting an exponential term containing the molecular positions and the total sample size $A=N_{\mathrm{c}} \mathcal{A}_{\mathrm{c}}$ of the semiconductor substrate given by the number of unit cells $N_{\mathrm{c}}$ and the unit cell area $\mathcal{A}_{\mathrm{c}}$ :

$$
\mathcal{V}_{\mathbf{k}^{\prime} \lambda^{\prime} B}^{\mathbf{k} \lambda} \equiv A e^{-\mathrm{i}\left(\mathbf{k}^{\prime}-\mathbf{k}\right) \cdot \mathbf{R}_{v}} V_{\mathbf{k}^{\prime} \lambda^{\prime} B_{v}}^{\mathbf{k} \lambda A_{v}}
$$

Note that the new quantity $\mathcal{V}_{\mathbf{k}^{\prime} \lambda^{\prime} B}^{\mathbf{k} \lambda}{ }_{A}^{A}$ does no longer depend on the molecular positions $\mathbf{R}_{v}$, reflecting the transformation of the molecular states to the Bloch basis representation. Section V and Appendix will provide further details on the derivation of the Coulomb matrix element.

\section{Equations of motion}

To calculate the absorption according to Eqs. (3) and (4), we now proceed to derive the equations of motion for the microscopic interband polarizations $\sigma_{\mathbf{k k}^{\prime}}^{\mathrm{vc}}=\left\langle a_{\mathbf{k}, \mathrm{v}}^{\dagger} a_{\mathbf{k}^{\prime}, \mathrm{c}}\right\rangle$ in the semiconductor and the microscopic molecular polarization $\sigma_{H L}^{\mathbf{l}, \mathbf{I}^{\prime}}=\left\langle a_{\mathbf{l}, H}^{\dagger} a_{\mathbf{I}^{\prime}, L}\right\rangle$ using the Heisenberg equation of motion. Focusing on the linear optical properties, the hierarchy problem arising from the correlation expansion of the Coulomb interaction is truncated at Hartree-Fock level [15,28,33,34], which yields an exact description of optical excitations in the linear optics limit [35]. Note that the molecular layer induces a spatially inhomogeneous charge distribution and thus we have to explicitly take into account polarizations with nonequal wave vectors $\mathbf{k} \neq \mathbf{k}^{\prime}$. For the polarization of the semiconductor, we obtain

$$
\begin{aligned}
\mathrm{i} \hbar \frac{\mathrm{d}}{\mathrm{d} t} \sigma_{\mathrm{vc}}^{\mathbf{k}, \mathbf{k}^{\prime}}= & \left(E_{\mathrm{c}}^{\mathbf{k}^{\prime}}-E_{\mathrm{v}}^{\mathbf{k}}\right) \sigma_{\mathrm{vc}}^{\mathbf{k}, \mathbf{k}^{\prime}}+\mathbf{E}(t) \cdot \mathbf{d}_{\mathrm{cv}}^{\mathbf{k}}\left(\rho_{\mathrm{v}}^{\mathbf{k}}-\rho_{\mathrm{c}}^{\mathbf{k}}\right) \delta_{\mathbf{k}, \mathbf{k}^{\prime}} \\
& +\frac{1}{A} \sum_{\mathbf{l}, \mathbf{I}^{\prime}} \sum_{\mathbf{G}} \mathcal{V}_{\mathbf{k v} L}^{\mathbf{k}^{\prime} \mathrm{c} H} \delta_{\mathbf{k}^{\prime}-\mathbf{k}, \mathbf{l}-\mathbf{l}^{\prime}+\mathbf{G}} \sigma_{H L}^{\mathbf{l}, \mathbf{I}^{\prime}}\left(\rho_{\mathrm{v}}^{\mathbf{k}}-\rho_{\mathrm{c}}^{\mathbf{k}^{\prime}}\right) .
\end{aligned}
$$

Similarly, we derive an equation of motion for the molecular polarization:

$$
\begin{aligned}
\mathrm{i} \hbar \frac{\mathrm{d}}{\mathrm{d} t} \sigma_{H L}^{\mathbf{l}, \mathbf{I}^{\prime}}= & \left(E_{L}-E_{H}\right) \sigma_{H L}^{\mathbf{l}, \mathbf{I}^{\prime}}+\mathbf{E}(t) \cdot \mathbf{d}_{L H}\left(\rho_{H}^{\mathbf{l}}-\rho_{L}^{\mathbf{l}}\right) \delta_{\mathbf{l}, \mathbf{I}^{\prime}} \\
& +\frac{1}{A} \sum_{\mathbf{k}_{1}, \mathbf{k}_{2}} \sum_{\mathbf{G}} \mathcal{V}_{H \mathbf{k}_{2} \mathrm{c}}^{L \mathbf{k}_{1} \mathrm{v}} \sigma_{\mathrm{vc}}^{\mathbf{k}_{1}, \mathbf{k}_{2}} \delta_{\mathbf{I}^{\prime}-\mathbf{l}, \mathbf{k}_{1}-\mathbf{k}_{2}+\mathbf{G}}\left(\rho_{H}^{\mathbf{l}}-\rho_{L}^{\mathbf{I}^{\prime}}\right) .
\end{aligned}
$$

\section{FÖRSTER TRANSFER MATRIX ELEMENTS}

The nonradiative coupling of the substrate to the molecular layer, Eqs. (17) and (18), is governed by the Coulomb matrix elements. In particular, the matrix elements $V_{H_{v} \mathbf{k}^{\prime} \mathrm{c}}^{L_{v} \mathbf{k v}}$ characterize the coherent coupling of the polarization of the $v$ th molecule to the interband polarization of the semiconductor substrate. To calculate the linear absorption spectra in the limit of low molecular coverage, no other matrix elements are needed. In the following, we will approximate these Coulomb matrix elements by applying a point-dipole approximation resulting in a dipole-dipole interaction between the semiconductor and the molecules. We will then use input from DFT to evaluate the resulting expression.

\section{A. Point-dipole approximation}

Generally, the Coulomb coupling matrix element is given by

$$
V_{H_{v} \mathbf{k}^{\prime} \mathrm{c}}^{L_{v} \mathbf{k v}}=\int \mathrm{d}^{3} r \int \mathrm{d}^{3} r^{\prime} \Psi_{L_{v}}^{*}(\mathbf{r}) \Psi_{\mathbf{k v}}^{*}\left(\mathbf{r}^{\prime}\right) V\left(\mathbf{r}-\mathbf{r}^{\prime}\right) \Psi_{H_{v}}\left(\mathbf{r}^{\prime}\right) \Psi_{\mathbf{k}^{\prime} \mathrm{c}}(\mathbf{r})
$$

with the Coulomb potential

$$
V\left(\mathbf{r}-\mathbf{r}^{\prime}\right)=\frac{e_{0}^{2}}{4 \pi \varepsilon_{0}} \frac{1}{\left|\mathbf{r}-\mathbf{r}^{\prime}\right|} .
$$

$\Psi_{A_{v}}(\mathbf{r})$ are the wave functions of molecular orbital $A \in$ $\{H, L\}$ on the $v$ th molecule and $\Psi_{\mathbf{k} \lambda}(\mathbf{r})$ the wave functions of a semiconductor states with wave vector $\mathbf{k}$ and band index $\lambda$. Approximating the interaction potential by a Taylor series around the molecule positions $\mathbf{R}_{v}$ and semiconductor elementary cells $\mathbf{R}_{\mathrm{V}}$ allows us to break up the double integral over all space into two separate integrals over each component (the molecular layer and the substrate) [36]. For details see Appendix. In the point-dipole approximation, we then obtain a closed analytical form of the Förster transfer element:

$$
\begin{aligned}
& V_{H_{\nu} \mathbf{k}+\mathbf{q} \mathrm{c}}^{L_{v} \mathbf{k} \mathrm{v}}=\frac{1}{A} \frac{e_{0}^{2}}{2 \varepsilon_{0}} e^{\mathrm{i} \mathbf{q} \cdot \mathbf{R}_{v}^{\|}} \frac{e^{-|\mathbf{q}| \Delta z}}{|\mathbf{q}|}\left[\left(\begin{array}{c}
q_{x} \\
q_{y} \\
-\mathrm{i}|\mathbf{q}|
\end{array}\right) \cdot \mathbf{d}_{L H}\right] \\
& \times\left[\left(\begin{array}{c}
q_{x} \\
q_{y} \\
-\mathrm{i}|\mathbf{q}|
\end{array}\right) \cdot \mathbf{d}_{\mathbf{k}, \mathbf{k}+\mathbf{q}}^{\mathrm{vc}}\right],
\end{aligned}
$$

where $\mathbf{d}_{\mathbf{k} \mathbf{k}^{\prime}}^{\lambda \lambda^{\prime}}$ are the transition dipoles from state $\lambda$ at $\mathbf{k}$ to state $\lambda^{\prime}$ at $\mathbf{k}^{\prime}$. In this study, we will neglect the wave vector dependence of $\mathbf{d}_{\mathbf{k} \mathbf{k}^{\prime}}^{\lambda \lambda^{\prime}}$, which is a good approximation close to the band edges, and take the value at the $\Gamma$ point (i.e., $\mathbf{d}_{\mathbf{k}, \mathbf{k}+\mathbf{q}}^{\mathrm{vc}} \approx \mathbf{d}_{\Gamma, \Gamma}^{\mathrm{vc}} \equiv \mathbf{d}^{\mathrm{vc}}$ ).

Figure 2 illustrates how the orientation of the dipole moments affects the transfer element in Eq. (21). For a parallel orientation of $\mathbf{d}^{\mathrm{vc}}$ and $\mathbf{d}^{H L}$, its maximal values lie along the axis of the dipole moments. The contours of equal value form a dumbbell-shaped pattern around the dipol orientation. When the dipoles are perpendicular to each other the dumbbell lobes split into four lobes oriented along the diagonals between the perpendicular dipole orientations. For a parallel orientation in the $z$ direction, the maximal value is found on a circle around the origin of the $\left(q_{x}, q_{y}\right)$-plane.

\section{LINEAR OPTICAL ABSORPTION SPECTRA}

\section{A. Solution in frequency space}

Equations (17) and (18) form a closed set of equations for the microscopic polarizations in the semiconductor substrate 

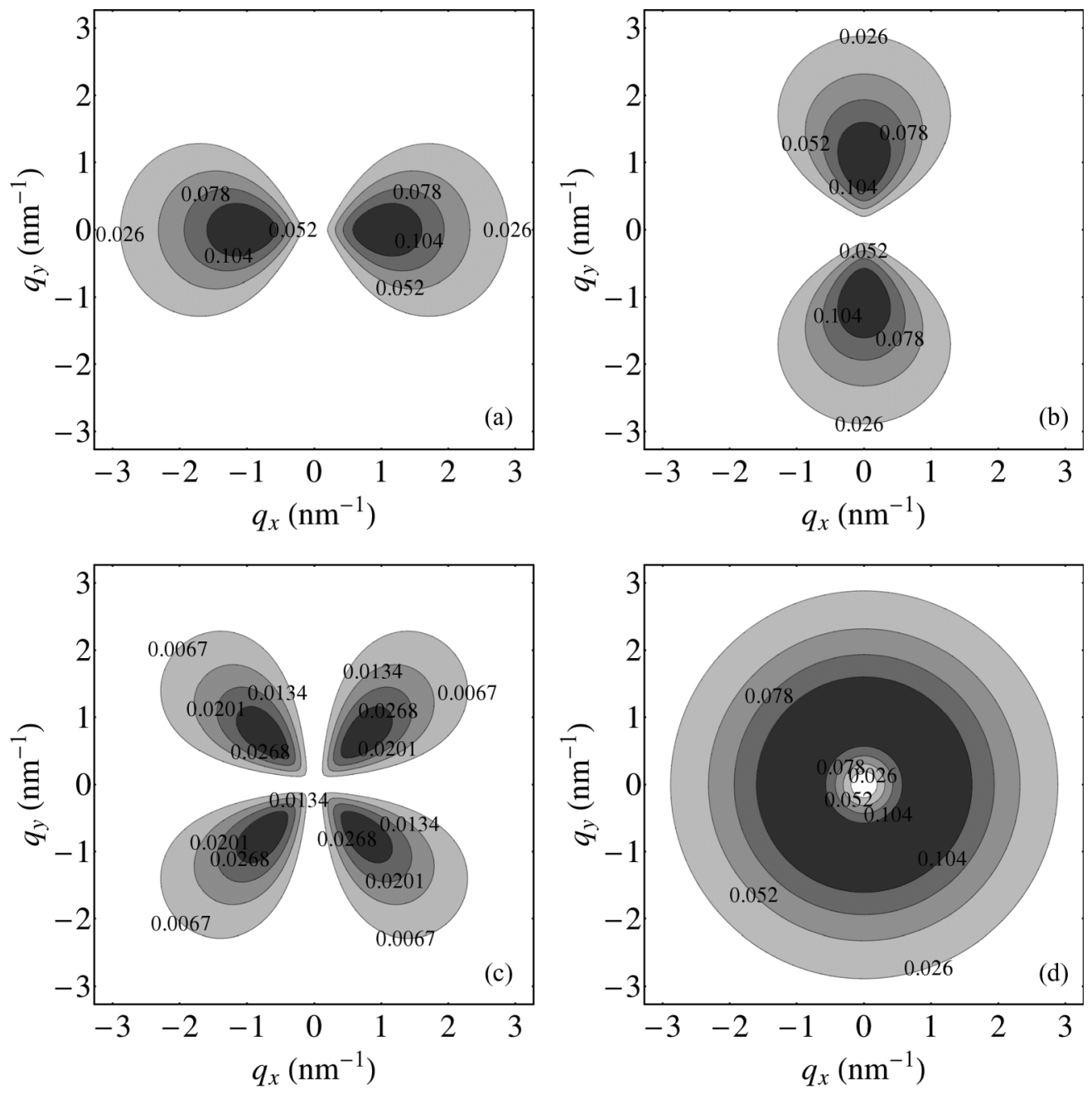

FIG. 2. Contour plot illustrating the interaction strength as given by the Förster transfer element $\mid V_{H_{v} \mathbf{k}+\mathbf{k} \mathrm{c}}^{L_{v} \mathbf{v}}{ }^{2}$ for (a) parallel dipoles in the $x$ direction, (b) parallel dipoles in the $y$ direction, (c) orthogonal dipoles (each one parallel to a coordinate axis), and (d) parallel dipoles in the $z$ direction.

and the molecular layer. In the following, we show how this system of equations can be solved in frequency space. First, we Fourier transform all quantities appearing in Eqs. (17) and (18): $\hat{f}(\omega)=\int f(t) e^{\mathrm{i} \omega t} \mathrm{~d} t$. Since we are only interested in weak optical excitations, we can assume the occupation densities $\rho_{H}^{\mathbf{1}}, \rho_{L}^{\mathbf{1}}, \rho_{\mathrm{v}}^{\mathbf{k}}$ and $\rho_{\mathrm{v}}^{\mathbf{k}}$ in Eqs. (17) and (18) to be constant in time. We apply the Fourier transform to Eqs. (17) and (18) and obtain

$$
\begin{aligned}
& \hat{\sigma}_{H L}^{\mathrm{l}, \mathrm{I}^{\prime}}(\omega)=\left[\hbar \omega-\left(E_{L}-E_{H}\right)+\mathrm{i} \gamma_{\mathrm{m}}\right]^{-1} \\
& \times\left\{\hat{\mathbf{E}}(\omega) \cdot \mathbf{d}_{L H}\left(\rho_{H}^{\mathbf{l}}-\rho_{L}^{\mathbf{l}}\right) \delta_{\mathbf{l}, \mathbf{I}^{\prime}}\right. \\
& \left.+\frac{1}{A} \sum_{\substack{\mathbf{k}_{1}, \mathbf{k}_{2} \\
\mathbf{G}_{2}}} V_{H \mathbf{k}_{2} \mathrm{c}}^{L \mathbf{k}_{1} \mathrm{v}} \hat{\sigma}_{\mathrm{vc}}^{\mathbf{k}_{1}, \mathbf{k}_{2}} \delta_{\mathbf{I}^{\prime}-\mathbf{l}, \mathbf{k}_{1}-\mathbf{k}_{2}+\mathbf{G}}\left[\rho_{H}^{1}-\rho_{L}^{I^{\prime}}\right]\right\}
\end{aligned}
$$

as well as

$$
\begin{aligned}
\hat{\sigma}_{\mathrm{vc}}^{\mathbf{k}, \mathbf{k}^{\prime}}(\omega)= & {\left[\hbar \omega-\left(E_{\mathrm{c}}^{\mathbf{k}^{\prime}}-E_{\mathrm{v}}^{\mathbf{k}}\right)+\mathrm{i} \gamma_{\mathrm{s}}\right]^{-1} } \\
& \times\left\{\hat{\mathbf{E}}(\omega) \cdot \mathbf{d}_{\mathrm{cv}}^{\mathbf{k}}\left(\rho_{\mathrm{v}}^{\mathbf{k}}-\rho_{\mathrm{c}}^{\mathbf{k}}\right) \delta_{\mathbf{k}, \mathbf{k}^{\prime}}\right. \\
& \left.+\frac{1}{A} \sum_{\substack{\mathrm{l} \mathbf{I}^{\prime} \\
\mathbf{G}}} V_{\mathbf{k v L}}^{\mathbf{k}^{\prime} \mathrm{c} H} \delta_{\mathbf{k}^{\prime}-\mathbf{k}, \mathbf{l}-\mathbf{I}^{\prime}+\mathbf{G}} \hat{\sigma}_{H L}^{\mathbf{l}, I^{\prime}}(\omega)\left[\rho_{\mathrm{v}}^{\mathbf{k}}-\rho_{\mathrm{c}}^{\mathbf{k}^{\prime}}\right]\right\} .
\end{aligned}
$$

Here we introduce the phenomenological linewidths $\gamma_{\mathrm{m}}$ and $\gamma_{\mathrm{s}}$ for transitions in the molecular layer and the semiconductor, respectively. They account for incoherent dephasing processes which are beyond the scope of this work.

As our model does not take into account any direct interaction between different molecules, all contributions to the polarization that arise from states located on different molecules vanish, i.e., $\left\langle a_{H v}^{\dagger} a_{L v^{\prime}}\right\rangle=\delta_{v, v^{\prime}}\left\langle a_{H v}^{\dagger} a_{L v}\right\rangle$. When we 
transform the molecular operators to the Bloch basis, we then find that the molecular polarization only depends on the difference and not the absolute values of the molecular wave vectors:

$$
\left\langle a_{H \mathbf{l}}^{\dagger} a_{L \mathbf{l}^{\prime}}\right\rangle=\frac{1}{N_{m}} \sum_{v} e^{\mathrm{i}\left(\mathbf{I}^{\prime}-\mathbf{l}\right) \cdot \mathbf{R}_{v}}\left\langle a_{H v}^{\dagger} a_{L v}\right\rangle .
$$

The computational effort is reduced, because only molecular polarizations with equal wave vectors $\left(\hat{\sigma}_{H L}^{\mathbf{l}, \mathbf{l}}(\omega)\right)$ are required for the calculation of the optical response in Eq. (4) $\left(\hat{\sigma}_{H L}^{1,1}(\omega) \equiv\right.$ $\left.\hat{\sigma}_{H L}^{\mathrm{l}}(\omega)\right)$. Although substrate polarizations with unequal wave vectors $\mathbf{k} \neq \mathbf{k}^{\prime}$ do not contribute to the optical far field response, as will be explained below, they are coupled to the molecular polarizations and thus need to be taken into account. To obtain a closed solution for the polarizations in frequency space, we combine Eqs. (22) and (23). To simplify the presentation, we first abbreviate the single-particle energy poles by

$$
X_{L H}(\omega) \equiv\left[\hbar \omega-\left(E_{L}-E_{H}\right)+\mathrm{i} \gamma_{\mathrm{m}}\right]^{-1}
$$

and

$$
Y_{\mathbf{k}, \mathbf{k}^{\prime}}(\omega) \equiv\left[\hbar \omega-\left(E_{\mathrm{c}}^{\mathbf{k}^{\prime}}-E_{\mathrm{v}}^{\mathbf{k}}\right)+\mathrm{i} \gamma_{\mathrm{s}}\right]^{-1} .
$$

The closed solution for the molecular polarization is given by

$$
\begin{aligned}
\hat{\sigma}_{H L}^{\mathbf{l}}(\omega)= & X_{L H}(\omega) \hat{\mathbf{E}}(\omega) \cdot \mathbf{d}_{L H}\left(\rho_{H}-\rho_{L}\right) \\
& \times\left[1-X_{L H}(\omega) \frac{N_{m}}{A^{2}} \sum_{\mathbf{k}_{1}} \sum_{\mathbf{G}}\left|\mathcal{V}_{H \mathbf{k}_{1}+\mathbf{G c}}^{L \mathbf{k}_{1} \mathrm{v}}\right|^{2}\right. \\
& \left.\times Y_{\mathbf{k}_{1}, \mathbf{k}_{1}+\mathbf{G}}(\omega)\left(\rho_{\mathrm{v}}^{\mathbf{k}_{1}}-\rho_{\mathrm{c}}^{\mathbf{k}_{1}+\mathbf{G}}\right)\left(\rho_{H}-\rho_{L}\right)\right]^{-1} .
\end{aligned}
$$

Note that in the above equation we have evaluated the Kronecker $\delta$ 's appearing in $H_{\mathrm{int}}$. To facilitate a cleaner notation, we have implicitly assumed that wave vectors $\mathbf{k}_{1}$ and $\mathbf{G}+\mathbf{k}_{1}$ are always from the first Brillouin zone of the semiconductor substrate. Accordingly, the corresponding sums have to be restricted in an appropriate manner.

\section{B. Special case: matching lattices}

To illustrate how the molecular coverage influences the optical properties of the hybrid, we take a look at the distribution of molecular reciprocal lattice vectors $\mathbf{G}$ in reciprocal space. The discussion is simplified by assuming the molecular lattice to match the surface lattice of the substrate. This implies that the molecular lattice vectors $\tilde{\mathbf{a}}_{1}$ and $\tilde{\mathbf{a}}_{2}$ become integer multiples of the substrate lattice vectors $\mathbf{a}_{1}$ and $\mathbf{a}_{2}$, which characterize the substrate surface, i.e., $\tilde{\mathbf{a}}_{i}=n_{i} \mathbf{a}_{i}$ with $n_{i} \in \mathbb{N}$. This reflects the fact that, in practice, the organic molecule is of greater spatial extent than the surface unit cell of the substrate and therefore one molecule covers many surface unit cells of the substrate. In the following, we will illustrate how the molecular arrangement affects the coupling strength by the example of a real world hybrid system, consisting of a $\mathrm{ZnO}(10 \overline{10})$ surface covered by a layer of ladder-type quarterphenyl (L4P) molecules [4,37]. The L4P molecule is

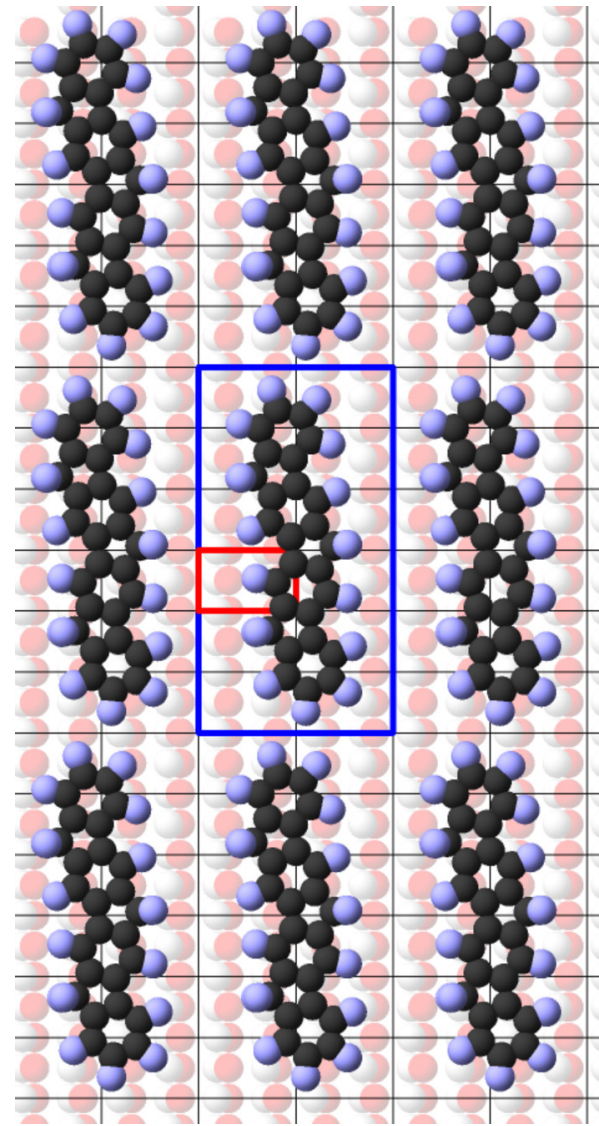

FIG. 3. (Color online) We demonstrate the relation between the substrate surface cell (red) and the molecular unit cell (blue) by means of a $\mathrm{L} 4 \mathrm{P}$ molecule on a $\mathrm{ZnO}(10 \overline{1} 0)$ surface.

an even-numbered ladder-type oligo( $p$-phenylene) (LOPP) providing favorable optoelectronic properties such as narrow absorption and luminescence bands as well as high absorption and luminescence coefficients [4]. Figure 3 demonstrates how one L4P molecule can be placed on a $\mathrm{ZnO}(10 \overline{10})$ surface covering 12 surface unit cells. The resulting molecular unit cell is then given by $2 \times 6$ cells of the substrate. While the molecular unit cell increases in real space with decreasing molecular coverage, the opposite is true in reciprocal space. Now the first Brillouin zone of the molecular layer is smaller than that of the substrate as we can verify by calculating the reciprocal lattice vectors $\mathbf{b}_{i}$ and $\tilde{\mathbf{b}}_{i}$. Using their definition, we find that $\tilde{\mathbf{b}}_{i}=\frac{1}{n_{i}} \mathbf{b}_{i}$, i.e., the size of the first Brillouin zone shrinks as the real space cell grows (see Fig. 4).

We can now asses the effect of the coverage and the molecular orientation on the Coulomb coupling strength. Equation (27) for the transfer matrix element tells us that $\mathbf{q}$ has to equal a reciprocal lattice vector $\mathbf{G}$, which is given as integer linear combination of the $\tilde{\mathbf{b}}_{i}$. Therefore the size of the $\tilde{\mathbf{b}}_{i}$ governs which points in reciprocal space contribute to the equation of motion (27). Figure 5 illustrates how different grids of reciprocal molecular lattice vectors $\mathbf{G}$ resulting from different molecular coverages can have a significant effect on the coupling strength. The left panel corresponds to coverage of one molecule per $2 \times 4$ substrate unit cells. The $\mathbf{G}$ vectors clearly miss the maxima of the Coulomb matrix element shown 

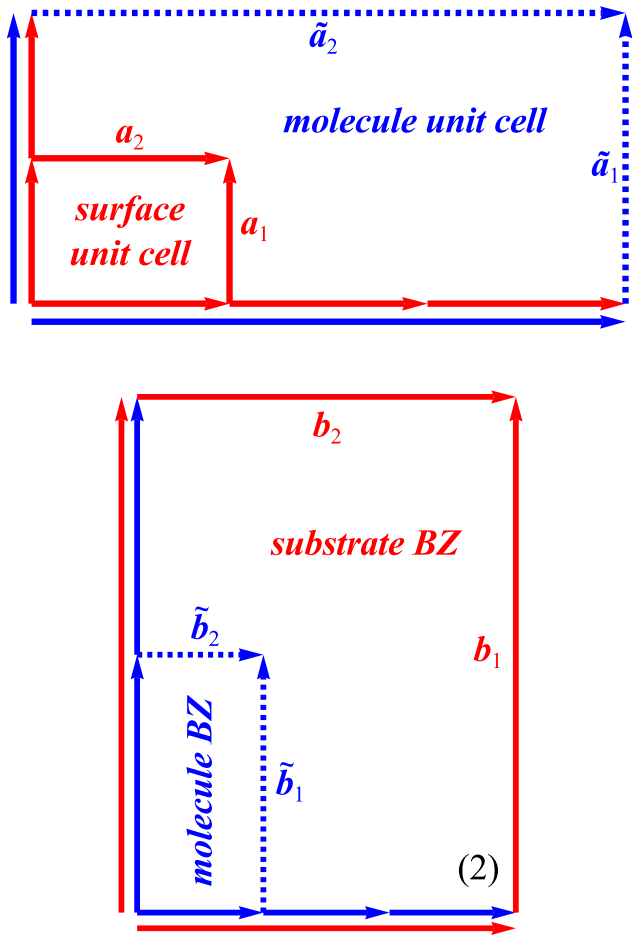

FIG. 4. (Color online) Illustration of real (1) vs reciprocal (2) space lattice by example of a $3 \times 2$ molecular layer.

by the contour plot. However, with decreasing molecular coverage, the density of the points reached by the $\mathbf{G}$ vectors increases and therefore the maxima of the coupling element are taken into account, leading to a stronger interaction. This reflects the fact that for larger coverages the oscillator strength of the semiconductor substrate is distributed among many molecules. The lower plot in Fig. 5 illustrates the position of the allowed momentum transfers $\mathbf{q}=\mathbf{G}$ for the situation where one molecule spans $10 \times 16$ surface unit cells

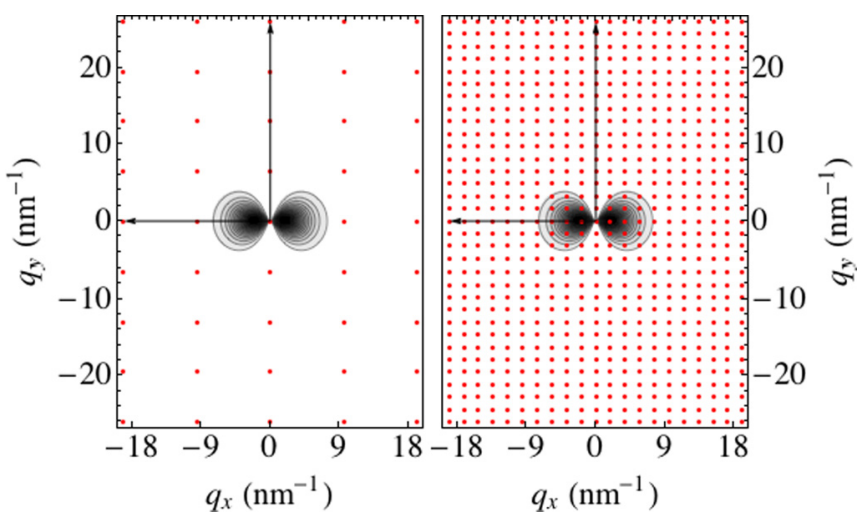

FIG. 5. (Color online) Red dots indicate the allowed momentum transfer wave vectors $\mathbf{q}$ for different molecular surface coverage densities. The underlying contour plot shows the Coulomb matrix element (see Fig. 2) The left figure clearly shows how the extrema of the Coulomb matrix element are missed for a $2 \times 4$ coverage. The right plot shows how the density of allowed momentum transfer vectors (i.e., $\mathbf{G}$ vectors in Eq. (27)) increases when the coverage is reduced to one molecule on $10 \times 16$ surface cells. of the substrate. Although our model does not include direct interactions between orbitals located on different molecules, the coupling to the substrate has a similar effect for finite distances between the molecules.

\section{NUMERICAL INVESTIGATION}

We now investigate the influence of the Förster coupling on the optical response of the hybrid layer. From Eq. (3), we see that the far field response does not take into account polarizations with different wave vectors $\mathbf{k} \neq \mathbf{k}^{\prime}$, which describe coherences on the microscopic spatial scale. On the other hand, Eq. (23) illustrates that the Förster interaction affects polarizations $\hat{\sigma}_{\mathrm{vc}}^{\mathbf{k}, \mathbf{k}^{\prime}}(\omega)$ with equal wave vectors $\mathbf{k}=\mathbf{k}^{\prime}$ only if the matrix element for zero momentum transfer $(\mathbf{q}=0)$ is nonvanishing. However, in the point-dipole approximation, the $\mathbf{q}=0$ element does vanish. As a result, the optical far field response of the substrate is not influenced by the molecular adlayer.

We will therefore not consider the optical spectrum of the semiconductor substrate and focus instead on the spectrum of the molecular film. To simplify our numerical investigation, we adopt the effective-mass approximation for the valence and conduction bands of $\mathrm{ZnO}$. The effective masses of $m_{\mathrm{c}}^{*}=1.4463 m_{0}$ for the conduction and $m_{\mathrm{v}}^{*}=-8.3035 m_{0}$ for the valence band have been obtain from a fit to the DFT band structure of bulk ZnO. All DFT calculations in this work have been performed with the Fritz-Haber-Institut $a b$ initio molecular simulations (FHI-aims) code [38,39] and the Heyd-Scuseria-Ernzerhof (HSE) [40,41] exchange-correlation functional. Following our previous work on $\mathrm{ZnO}$ [42], the exact-exchange admixture was chosen to be $40 \%$, which yields a band gap of $E_{g}=3.4 \mathrm{eV}$. As mentioned earlier, we adjust the HOMO-LUMO resonance of the molecular adlayer to be just above the substrate band gap at $E_{H L}=3.401 \mathrm{eV}$. The distance between the substrate surface and the molecular layer is fixed to $\Delta z=1 \mathrm{~nm}$. The substrate unit cell is given by the lattice vectors $\mathbf{a}_{1}=0.3289 \mathrm{~nm} \mathbf{e}_{x}$ and $\mathbf{a}_{2}=0.5242 \mathrm{~nm} \mathbf{e}_{y}$, where $\mathbf{e}_{x}$ and $\mathbf{e}_{y}$ denote vectors of unit length, spanning the substrate surface plane. In all calculations, the substrate dipole moment is fixed to point in the direction of $\mathbf{e}_{x}$ and its strength of $\left|\mathbf{d}_{\mathrm{cv}}\right|=0.012 e_{0} \mathrm{~nm}$ was calculated in HSE. The geometry of the L4P molecule was relaxed in the gas phase. The dipole strength for the molecular HOMO-LUMO transition amounts to $\left|\mathbf{d}_{H L}\right|=0.35 e_{0} \mathrm{~nm}$. The angle of the molecular transition dipole moment and $\mathbf{d}_{\mathrm{cv}}$ is given by the angle $\alpha$.

Figure 6 shows the resulting absorption spectra of the hybrid system for rectangular molecular unit cells of different sizes. Due to the narrow linewidth and the strong dipole moment, the single-particle spectra are referenced to that of an isolated molecule, which consists of a Lorentzian peak at the energy of the HOMO-LUMO transition energy. As the system is brought into contact with the substrate, the attractive Coulomb interaction between electrons and holes gives rise to a reduced optical transition energy which manifests itself by a redshifted absorption line in the absorption spectrum. As discussed earlier, the energy difference $\Delta E$ (see Fig. 6) depends on the size and the aspect ratio of the molecular unit cell. Figure 6 illustrates the red shift of the HOMO- 


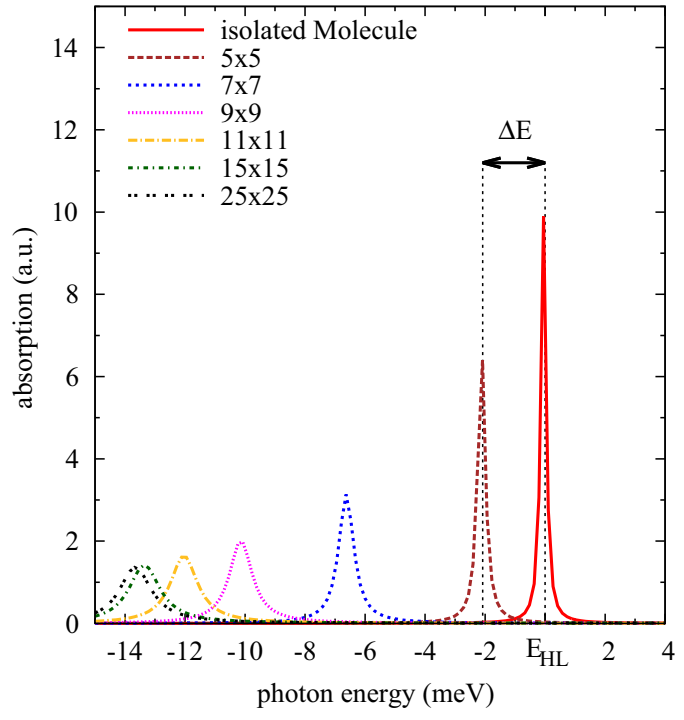

FIG. 6. (Color online) The energy shift $\Delta E$ depends on the molecular coverage density. The lines indicate molecular unit cells of varying size and fixed aspect ratio.

LUMO absorption line depending on the size of the molecular cell, as well as the decreasing intensity as the molecular coverage is decreasing. To investigate the dependence of the energy shift $\Delta E$ and the linewidth $\gamma$ on the geometry parameters $\alpha, \Delta z$ and the aspect ratio of the molecular unit cell, we fit the spectra with a Lorentzian. In Fig. 7, we show the energy shift in dependence of the molecular unit cell size for fixed aspect ratios. For small coverages (i.e., large molecular cells), the energy shift approaches a limiting value that is independent of the aspect ratio. For higher molecular coverages, the limiting value depends strongly on the aspect ratio of the molecular cell. Both effects can be explained by the

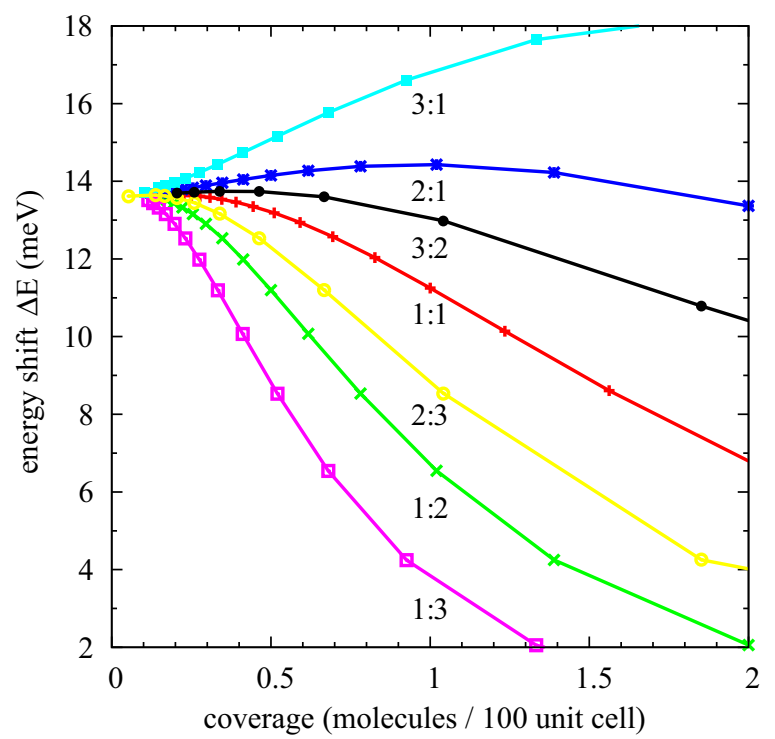

FIG. 7. (Color online) The energy shift $\Delta E$ depends on the molecular coverage. The lines indicate molecular unit cells of varying size and fixed aspect ratio for parallel dipole moments oriented along the $x$ axis.

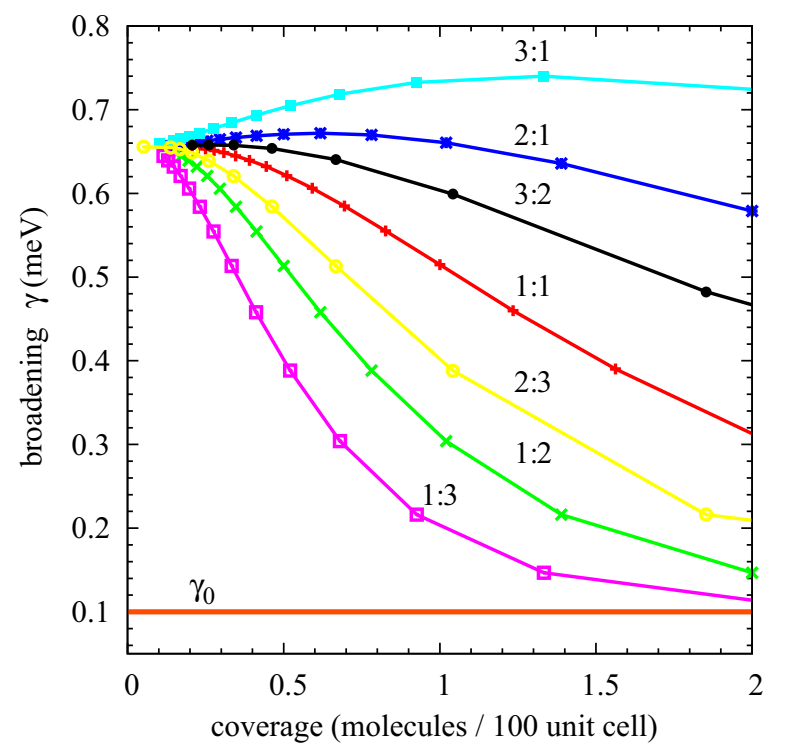

FIG. 8. (Color online) The fitted linewidth $\gamma$ as function of the molecular coverage for varying molecular unit cells of fixed aspect ratio.

aforementioned relation between real and reciprocal spaces. For decreasing molecular coverages, the density of $\mathbf{G}$ points in reciprocal space increases until it becomes quasi continuous, i.e., independent on the aspect ratio. The previous increases the effective interaction strength until it reaches the limiting value of the quasicontinuous density of $\mathbf{G}$ points. In this limit, the average distance between any two molecules becomes infinitely large. Therefore the geometry of the molecular unit cell (i.e., the position of the next neighboring molecule) has no influence on the optical response of the molecular layer. As can be seen from Fig. 7 the energy shift is especially strong for aspect ratios greater than 1, i.e., the unit cell has a larger extent in the $x$ direction, which coincides with the orientation of the dipole moments. Accordingly, if the aspect ratio is less than 1 , and therefore perpendicular to the orientation of the dipole moments, the interaction is weak. This behavior is linked to the density of allowed momentum transfer vectors (see Fig. 5). Different aspect ratios cause nonuniform densities of allowed momentum transfer vectors while the total coverage density $N_{m} / N_{\mathrm{c}}$ stays constant. When going towards the single-molecule limit (i.e., the left of Fig. 7) the molecular coverage decreases while the overall density of momentum transfer vectors increases. The interaction strength is governed by a counteraction of the total coverage density versus the increase of allowed transfer processes. For instance, in the 3:1 case, the density of allowed momentum transfer vectors along the dipole axis is higher than in the orthogonal direction. Here, the decrease in coverage outweighs the increase of allowed processes, leading to a decrease in energy shift towards lower coverage. The opposite situation occurs when the density of transfer processes along the dipole axis is lower (e.g., for a 1:3 aspect ratio). For higher molecular coverage, the density of $\mathbf{G}$ points is low and accordingly the effective interaction strength decreases.

Figure 8 shows the corresponding linewidth $\gamma$. In the figures, the phenomenological broadening $\gamma_{\mathrm{m}}$ of an isolated 


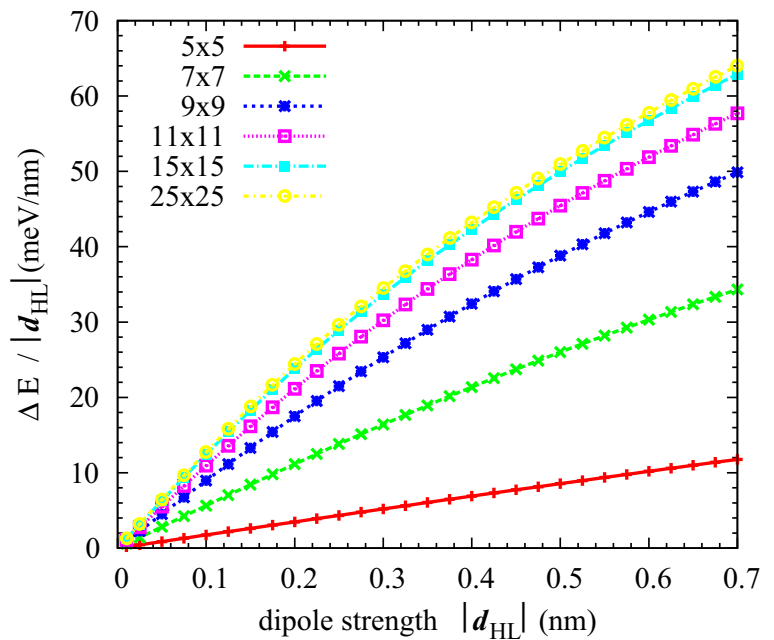

FIG. 9. (Color online) The energy shift of the HOMO-LUMO transition exhibits a quadratic dependence on the transition dipole that enters the Coulomb matrix element. The DFT-HSE value is $0.35 e_{0} \mathrm{~nm}$.

molecule introduced in Eq. (25) is referred to as $\gamma_{0}$. For high coverage (thus low coupling strength), the broadening approaches $\gamma_{0}$, the linewidth of the molecular resonance. We clearly find that the hybridization causes a significant contribution to the linewidth exhibiting a similar trend as the peak shift $\Delta E$. The additional broadening is attributed to the Coulomb interaction of the energetically sharp HOMOLUMO excitation with the energetically distributed interband excitations in the semiconductor, since the molecule polarizations can decay into a continuum of electronic semiconductor excitations. In Fig. 9 and 10, it can be seen, that both the energy shift and the linewidth increase for increasing dipole moment and thus coupling.

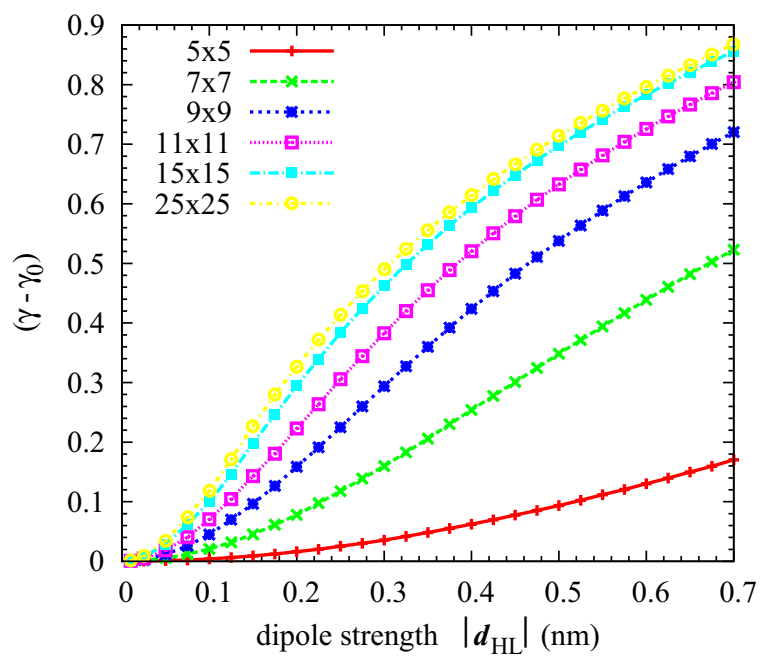

FIG. 10. (Color online) The linewidth caused by the hybridization (i.e., observed linewidth less the phenomenological linewidth $\gamma_{0}$ ) in dependence of the transition dipole.

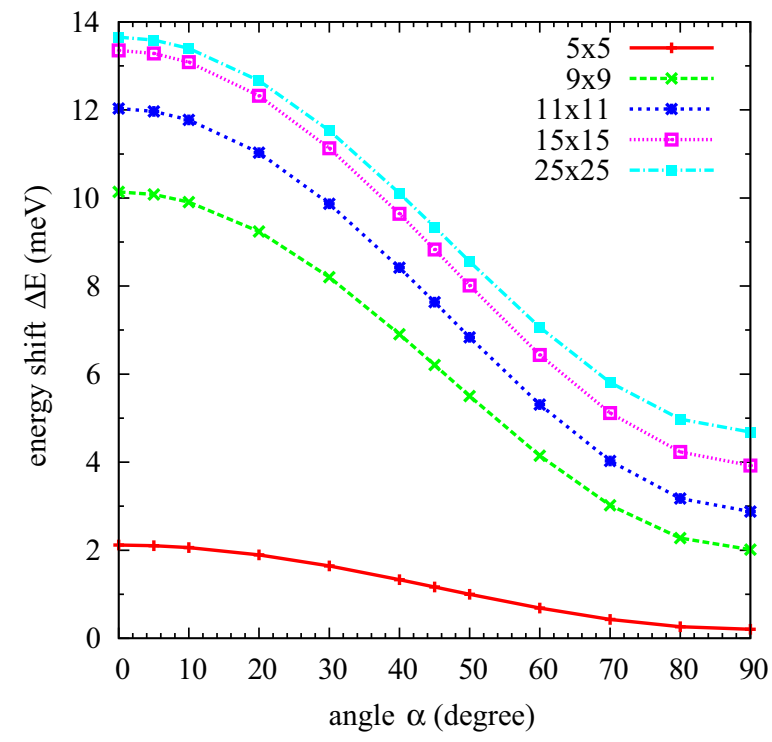

FIG. 11. (Color online) The energy shift of the HOMO-LUMO transition for various square molecular unit cells as a function of the relative dipole angle $\alpha$.

Finally, we show how the relative orientation of the dipole moments of the individual constituents affects the interaction strength. We therefore vary the orientation of the molecular transition dipole moment $\mathbf{d}_{H L}$ while the substrate transition dipole $\mathbf{d}_{\mathrm{vc}}$ remains constant. In Fig. 11, we plot the energy shift as a function of the angle $\alpha$ between $\mathbf{d}_{H L}$ and $\mathbf{d}_{\mathrm{cv}}$ for molecular cells of square aspect ratios (see Fig. 12 for a corresponding plot of the broadening). We find the strongest interaction for dipoles with parallel orientation at $\alpha=0^{\circ}$ and it decreases with a cosine dependence, reaching a finite nonzero minimum for perpendicular dipole moments.

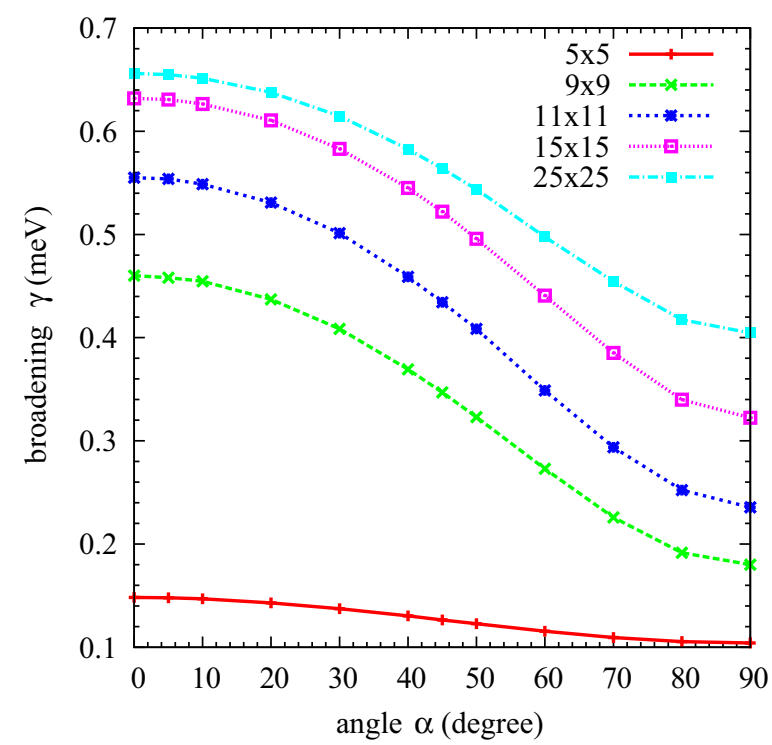

FIG. 12. (Color online) Dependence of the peak broadening on the relative dipole orientation. 


\section{CONCLUSION}

We developed optical Bloch equations for the coupling of molecular and semiconductor continuum states in hybrid inorganic-organic systems. The Förster-type Coulomb interaction couples the optical excitations in the organic and in the inorganic layer and results in combined excitations. This Förster-type interaction was included as a dipole-dipoleinteraction between the two HIOS components. To calculate optical absorption spectra, we solved the Bloch equations for the hybrid system. We then tested the sensitivity of the Förster interaction strength to the geometric arrangement of the molecules in the molecular layer relative to the inorganic substrate. At lower molecular coverages, the interaction becomes stronger than at higher coverage since in that case the oscillator strength of the semiconductor is distributed among more molecules. We found that the HOMOLUMO transition line is shifted towards lower energies for increasing interaction strength. Our calculations also revealed that and how the interaction and therefore the energy shift is maximized for parallel transition dipole moments. Although the intermolecular Coulomb coupling was neglected in this work, it may play an important role for the formation of localized excitons in the organic layer, enhancing the Förster coupling and will be included in future work. Though the point-dipole approximation yields a straightforward approach of reduced complexity towards the complex problem of the interlayer Coulomb coupling, the material systems may be more accurately modeled by going beyond the point-dipole approximation for the Coulomb interaction using, e.g., the partial charge technique [43]. Furthermore, we have seen very specific selection rules regarding the Coulomb coupling. In conclusion, tuning the coverage density and geometry is crucial to obtain optimum performance of hybrid devices used in photovoltaic or similar optoelectronic applications. It is likely, that the presented framework can provide valuable guidance in geometry optimization, if extended by, e.g., more detailed coupling elements.

\section{ACKNOWLEDGMENT}

We acknowledge financial support from the DFG through SFB 951 Hybrid Inorganic/Organic Systems for OptoElectronics (HIOS).

\section{APPENDIX: FÖRSTER TRANSFER MATRIX ELEMENT}

This section provides additional details to the calculation of the Förster transfer element in the dipole approximation [36]. Generally, the Coulomb coupling matrix element is given by

$$
\begin{aligned}
V_{H_{\nu} \mathbf{k}^{\prime} \mathrm{c}}^{L_{\nu} \mathbf{k v}}= & \int \mathrm{d}^{3} r \int \mathrm{d}^{3} r^{\prime} \Psi_{L_{v}}^{*}(\mathbf{r}) \Psi_{\mathbf{k v}}^{*}\left(\mathbf{r}^{\prime}\right) \\
& \times V\left(\mathbf{r}-\mathbf{r}^{\prime}\right) \Psi_{H_{v}}\left(\mathbf{r}^{\prime}\right) \Psi_{\mathbf{k}^{\prime} \mathrm{c}}(\mathbf{r}) .
\end{aligned}
$$

By approximating the interaction potential

$$
\begin{aligned}
V\left(\mathbf{r}-\mathbf{r}^{\prime}\right) & =\frac{e_{0}^{2}}{4 \pi \varepsilon_{0}} \frac{1}{\left|\mathbf{r}-\mathbf{r}^{\prime}\right|} \\
& \approx \frac{e_{0}^{2}}{4 \pi \varepsilon_{0}}\left[\frac{\tilde{\mathbf{r}} \cdot \tilde{\mathbf{r}}^{\prime}}{|\mathcal{R}|^{3}}-3 \frac{(\mathcal{R} \cdot \tilde{\mathbf{r}})\left(\mathcal{R} \cdot \tilde{\mathbf{r}}^{\prime}\right)}{|\mathcal{R}|^{5}}\right]
\end{aligned}
$$

by a Taylor series, we can factorize the nested integration. The expansion is based around the molecule positions $\mathbf{R}_{v}$ and the substrate lattice vectors pointing to the unit cell positions $\mathbf{R}_{\mathrm{V}}$ respectively. We define the vector $\mathcal{R}=\mathbf{R}_{\mathrm{V}}-\mathbf{R}_{v}$ as their difference and obtain

$$
\begin{aligned}
V_{H_{v} \mathbf{k}^{\prime} \mathrm{c}}^{L_{v} \mathbf{k v}}= & \frac{e_{0}^{2}}{4 \pi \varepsilon_{0}} \sum_{\mathbf{R}_{\mathrm{v}}}^{N_{\mathrm{c}}} \int_{\mathcal{V}_{c}} \mathrm{~d}^{3} \tilde{r} \int_{\mathcal{V}_{m}} \mathrm{~d}^{3} \tilde{r}^{\prime} \Psi_{\mathbf{k v}}^{*}\left(\mathbf{R}_{\mathrm{V}}+\tilde{\mathbf{r}}\right) \\
& \times \Psi_{\mathbf{k}^{\prime} \mathrm{c}}\left(\mathbf{R}_{\mathrm{V}}+\tilde{\mathbf{r}}\right) \Psi_{L}^{*}\left(\mathbf{R}_{v}+\tilde{\mathbf{r}}^{\prime}\right) \Psi_{H}\left(\mathbf{R}_{v}+\tilde{\mathbf{r}}^{\prime}\right) \\
& \times\left[\frac{\tilde{\mathbf{r}} \cdot \tilde{\mathbf{r}}^{\prime}}{|\mathcal{R}|^{3}}-3 \frac{(\mathcal{R} \cdot \tilde{\mathbf{r}})\left(\mathcal{R} \cdot \tilde{\mathbf{r}}^{\prime}\right)}{|\mathcal{R}|^{5}}\right] .
\end{aligned}
$$

The integrals over the individual constituents wave functions can now be evaluated independently, allowing the introduction of the transition dipole moments. The wave functions in the semiconductor substrate are given by Bloch-functions in the in-plane direction $\mathbf{r}_{\|}$. The confinement in the perpendicular direction $(z)$ is taken into account by making the envelope approximation with the envelope function $\xi(z)$ :

$$
\Psi_{\mathbf{k} \lambda}(\mathbf{r})=\frac{1}{\sqrt{A}} e^{\mathrm{i} \mathbf{k} \cdot \mathbf{r}} u_{\mathbf{k} \lambda}\left(\mathbf{r}_{\|}\right) \xi(z) .
$$

With the transition dipole moment defined by

$\mathbf{d}_{\mathbf{k} \mathbf{k}^{\prime}}^{\lambda \lambda^{\prime}}=\frac{e_{0}}{\mathcal{A}_{\mathrm{c}}} \int_{-L / 2}^{L / 2} \mathrm{~d} z \int_{\mathcal{A}_{\mathrm{c}}} \mathrm{d}^{2} r_{\|} u_{\mathbf{k} \lambda}^{*}\left(\mathbf{r}_{\|}\right) \mathbf{r} u_{\mathbf{k}^{\prime} \lambda^{\prime}}\left(\mathbf{r}_{\|}\right) e^{\mathrm{i}\left(\mathbf{k}^{\prime}-\mathbf{k}\right) \cdot \mathbf{r}}|\xi(z)|^{2}$,

we can write the matrix element as

$$
\begin{aligned}
V_{H_{v}}^{L_{v} \mathbf{k v} \mathrm{c}}= & \frac{1}{4 \pi \varepsilon_{0}} \frac{1}{N_{\mathrm{c}}} e^{\mathrm{i}\left(\mathbf{k}^{\prime}-\mathbf{k}\right) \cdot \mathbf{R}_{v}} \sum_{\mathbf{R}_{\mathrm{v}}}^{N_{\mathrm{c}}} e^{\mathrm{i}\left(\mathbf{k}^{\prime}-\mathbf{k}\right) \cdot \mathcal{R}} \\
& \times\left[\frac{\mathbf{d}_{\mathbf{k} \mathbf{k}^{\prime}}^{\mathrm{vc}} \cdot \mathbf{d}_{L H}^{v}}{|\mathcal{R}|^{3}}-3 \frac{\left(\mathcal{R} \cdot \mathbf{d}_{\mathbf{k k}^{\prime}}^{\mathrm{vc}}\right)\left(\mathcal{R} \cdot \mathbf{d}_{L H}^{v}\right)}{|\mathcal{R}|^{5}}\right] .
\end{aligned}
$$

Since the substrate is a quasi-two-dimensional system, the wave vector $\mathbf{k}$ is restricted to a two-dimensional plane corresponding to the reciprocal space of the substrate surface. Therefore all scalar products in Eq. (A6) project the molecular position to the substrate surface. However, the norm of $\mathcal{R}$ still includes the surface-to-molecule distance $\Delta z$, i.e.,

$$
|\mathcal{R}|=\left(\left|\mathbf{R}_{V}^{\|}-\mathbf{R}_{\nu}^{\|}\right|^{2}+\Delta z^{2}\right)^{1 / 2} .
$$

Under the assumption, that the spatial extent of a substrate unit cell is small in comparison to the distance $\Delta z$, we can approximate the sum over all unit cells as an integral:

$$
\sum_{\mathbf{R}_{\mathrm{c}}}^{N_{\mathrm{c}}} \rightarrow \frac{1}{\mathcal{A}_{\mathrm{c}}} \int_{\mathbb{R}^{2}} \mathrm{~d}^{2} r
$$

By carrying out the integration, we obtain a closed analytical form of the Förster transfer element:

$$
\begin{aligned}
& V_{H_{\nu} \mathbf{k}+\mathbf{q} \mathrm{c}}^{L_{\nu} \mathbf{k} \mathrm{v}}=\frac{1}{A} \frac{e_{0}^{2}}{2 \varepsilon_{0}} e^{\mathrm{i} \mathbf{q} \cdot \mathbf{R}_{v}^{\|}} \frac{e^{-|\mathbf{q}| \Delta z}}{|\mathbf{q}|}\left[\left(\begin{array}{c}
q_{x} \\
q_{y} \\
-\mathrm{i}|\mathbf{q}|
\end{array}\right) \cdot \mathbf{d}_{L H}\right] \\
& \times\left[\left(\begin{array}{c}
q_{x} \\
q_{y} \\
-\mathrm{i}|\mathbf{q}|
\end{array}\right) \cdot \mathbf{d}_{\mathbf{k}, \mathbf{k}+\mathbf{q}}^{\mathrm{vc}}\right] \text {. }
\end{aligned}
$$


[1] D. Basko, G. C. La Rocca, F. Bassani, and V. M. Agranovich, Euro. Phys. J. B 8, 353 (1999).

[2] A. A. R. Neves, A. Camposeo, R. Cingolani, and D. Pisignano, Adv. Funct. Mater. 18, 751 (2008).

[3] T. Liang, Y. Cui, J. Yu, W. Lin, Y. Yang, and G. Qian, Thin Solid Films 544, 407 (2013).

[4] B. Kobin, L. Grubert, S. Blumstengel, F. Henneberger, and S. Hecht, J. Mater. Chem. 22, 4383 (2012).

[5] V. Agranovich, R. Atanasov, and F. Bassani, Solid State Commun. 92, 295 (1994).

[6] V. M. Agranovich, D. M. Basko, G. C. L. Rocca, and F. Bassani, J. Phys.: Condens. Matter 10, 9369 (1998).

[7] G. Itskos, G. Heliotis, P. G. Lagoudakis, J. Lupton, N. P. Barradas, E. Alves, S. Pereira, I. M. Watson, M. D. Dawson, J. Feldmann, R. Murray, and D. D. C. Bradley, Phys. Rev. B 76, 035344 (2007).

[8] S. Blumstengel, S. Sadofev, C. Xu, J. Puls, and F. Henneberger, Phys. Rev. Lett. 97, 237401 (2006).

[9] D. Hofmann, T. Körzdörfer, and S. Kümmel, Phys. Rev. A 82, 012509 (2010).

[10] X. Zhang, Z. Li, and G. Lu, Phys. Rev. B 84, 235208 (2011).

[11] W. Mou, S. Ohmura, F. Shimojo, and A. Nakano, Appl. Phys. Lett. 100, 203306 (2012).

[12] F. Shimojo, S. Ohmura, W. Mou, R. K. Kalia, A. Nakano, and P. Vashishta, Comp. Phys. Commun. 184, 1 (2013).

[13] C. A. Rozzi, S. M. Falke, N. Spallanzani, A. Rubio, E. Molinari, D. Brida, M. Maiuri, G. Cerullo, H. Schramm, J. Christoffers, and C. Lienau, Nat. Commun. 4, 1602 (2013).

[14] T. Förster, Ann. Phys. (Berlin) 437, 55 (1948).

[15] M. Lindberg and S. W. Koch, Phys. Rev. B 38, 3342 (1988).

[16] F. Rossi and T. Kuhn, Rev. Mod. Phys. 74, 895 (2002).

[17] H. Haug and S. Koch, Quantum Theory of the Optical and Electronic Properties of Semiconductors, 5th ed. (World Scientific, Singapore, 2009).

[18] M. Kira and S. W. Koch, Semiconductor Quantum Optics (Cambridge University Press, Cambridge, UK, 2012).

[19] T. Renger and V. May, J. Phys. Chem. B 101, 7232 (1997).

[20] T. Meier, V. Chernyak, and S. Mukamel, J. Chem. Phys. 107, 8759 (1997).

[21] T. Renger and R. A. Marcus, J. Chem. Phys. 116, 9997 (2002).
[22] T. Mančal, A. V. Pisliakov, and G. R. Fleming, J. Chem. Phys. 124, 234504 (2006).

[23] F. Šanda and S. Mukamel, Phys. Rev. Lett. 98, 080603 (2007).

[24] E. Malic, C. Weber, M. Richter, V. Atalla, T. Klamroth, P. Saalfrank, S. Reich, and A. Knorr, Phys. Rev. Lett. 106, 097401 (2011).

[25] M. Schoth, M. Richter, A. Knorr, and T. Renger, Phys. Rev. Lett. 108, 178104 (2012).

[26] D. Abramavicius, A. Nemeth, F. Milota, J. Sperling, S. Mukamel, and H. F. Kauffmann, Phys. Rev. Lett. 108, 067401 (2012).

[27] D. Abramavicius, Europhys. Lett. 101, 57007 (2013).

[28] M. Kira and S. Koch, Prog. Quant. Electron. 30, 155 (2006).

[29] L. Hedin, Phys. Rev. 139, A796 (1965).

[30] G. Onida, L. Reining, and A. Rubio, Rev. Mod. Phys. 74, 601 (2002).

[31] P. Rinke, A. Qteish, J. Neugebauer, C. Freysoldt, and M. Scheffler, New J. Phys. 7, 126 (2005).

[32] J. C. Slater, Rev. Mod. Phys. 6, 209 (1934).

[33] V. Axt and A. Stahl, Z. Phys. B 93, 195 (1994).

[34] J. Fricke, Ann. Phys. 252, 479 (1996).

[35] K. Victor, V. Axt, G. Bartels, A. Stahl, K. Bott, and P. Thomas, Z. Phys. B 99, 197 (1995).

[36] J. Danckwerts, K. J. Ahn, J. Förstner, and A. Knorr, Phys. Rev. B 73, 165318 (2006).

[37] H.-H. Huang, C. Prabhakar, K.-C. Tang, P.-T. Chou, G.-J. Huang, and J.-S. Yang, J. Am. Chem. Soc. 133, 8028 (2011).

[38] V. Blum, F. Hanke, R. Gehrke, P. Havu, V. Havu, X. Ren, K. Reuter, and M. Scheffler, Comp. Phys. Commun. 180, 2175 (2009).

[39] X. Ren, P. Rinke, V. Blum, J. Wieferink, A. Tkatchenko, A. Sanfilippo, K. Reuter, and M. Scheffler, New J. Phys. 14, 053020 (2012).

[40] J. Heyd, G. E. Scuseria, and M. Ernzerhof, J. Chem. Phys. 118, 8207 (2003).

[41] J. Heyd, G. E. Scuseria, and M. Ernzerhof, J. Chem. Phys. 124, 219906 (2006).

[42] N. Moll, Y. Xu, O. T. Hofmann, and P. Rinke, New J. Phys. 15, 083009 (2013).

[43] M. E. Madjet, A. Abdurahman, and T. Renger, J. Phys. Chem. B 110, 17268 (2006). 